\title{
Temperature Development in the Leakage Flow of Screw Extruders
}

\author{
H. E. H. MEYER, J. F. INGEN HOUSZ, and W. C. M. GORISSEN \\ Twente University of Technology \\ Plastics Processing Laboratory \\ Enschede, The Netherlands
}

\begin{abstract}
Temperature distribution at the exit of the leakage gap is of interest for a number of problems. For the calculation of temperatures, the leakage flow may be considered to be a pure drag flow to a good approximation. In the Newtonian case, thermal development length may be expressed in terms of gap height as $L \approx 3 / 8 P e \cdot \delta$; usually this is less than the available gap length.Pe is the Peclet number and $\delta$ the height of leakage gap. Therefore the existing flow may be considered fully developed. For power law fluids, numerical calculations lead to results of the same order. Martin's results therefore may be applied to the flow at the exit of the leakage gap.
\end{abstract}

\section{INTRODUCTION}

$\mathrm{K}$ nowledge of the temperature distribution in the leakage flow of a single screw extruder, at the place where this flow leaves the gap between screw flight and barrel and enters the main flow in the channel, is required for the solution of a number of problems.

Among these are calculations of the bulk mean temperature as it develops in the main channel and the determination of the temperature distribution in this main flow. As the leakage flow rate is usually not more than 10 percent of the extruder throughput an estimate of the mean temperature of the leakage flow to within a few degrees centigrade is sufficiently accurate for this purpose. A problem requiring a more accurate estimate, not only of the mean temperature but preferably also of the temperature distribution in the leakage flow at exit from the gap, is the calculation of the melting rate, using a refined extension of the model introduced by Tadmor (1), (improved a.o. by Shapiro (2) and by Fenner \& Edmondson (3)), by taking into account the convection terms in the calculation of the flow in the melt film.

In principle the problem was solved by Yates (4), who uses a set of three (simultaneous) coupled differential equations to describe momentum and heat transfer in the leakage gap. The numerical solution of these equations requires a significant amount of computer time. In cases where changing or developing boundary conditions along the channel length require the repeated use of this solution, which is the case in the modelling of the melting process, the time involved becomes prohibitive. It is a purpose of this work to develop a model which is sufficiently economic of computer time to allow its incorporation as a subroutine in an integrated computer simulation of a single screw extruder.

\section{LEAKAGE FLOW}

Leakage effects have been included in the analysis of the metering zone of single screw extruders since the first theories for this zone were developed. Notably Mohr \& Mallouk (5), using mass balances, gave a very accurate and complete analysis of the isothermal Newtonian case in the oblique channel approximation. By a completely different approach, Ingen Housz (6), using a method developed by Muyderman in his work on spiral groove bearings (7), reaches an identical result. The latter method, repeated in cylindrical coordinates, shows that the influence of channel curvature on the magnitude of the leakage flow is negligible. For a screw having a relatively small clearance in the barrel and operating with an axial pressure gradient (positive or negative) within the range encountered in practice, the above results show that the velocity profile in the leakage gap is only marginally influenced by the pressure gradient over the gap. This conclusion finds some experimental support in the work of Holmes (8) who used corn syrup in his experiments. Consequently the flow of a Newtonian fluid in the leakage gap may, as a rule, be treated as a pure drag flow.

For a power law fluid the influence of the pressure gradient may be much larger. Hayashida (9) considered this case but his results are not practical for a quick evaluation. In Ref. (6), an equation is given which allows the use of the ratio of estimated average viscosities in channel and gap to be applied to this problem. For a rather extreme ratio the pressure gradient may cause an increase of the leakage flow over the pure drag flow of up to 50 percent for otherwise normal conditions. The leakage flow calculated in this manner is a flow perpendicular to the screw flight, i.e., it is equal to the transverse 
component of flow in the channel. In the clearance the component of drag flow parallel to the flight direction is roughly three times the transverse component, while the influence of the pressure gradient in this direction is considerably smaller than in the transverse direction.

As a result, the velocity vector in the leakage gap is increased by not more than 10 percent on average under extreme conditions. Normally therefore temperature development in the clearance may be considered to depend on drag flow only. Even in cases where the pressure gradient contributes significantly to the leakage flowrate it may be neglected without materially affecting the calculated temperatures.

By this simplification the problem of determining velocity and temperature profiles at the exit side of the clearance reduces to a problem of drag flow between parallel flat plates. This was solved by Martin (10) for a power law fluid with temperature dependent viscosity. Martin however uses infinitely extending plates while the leakage gap is relatively short in the flow direction. The plate separation is very small and it is therefore not unreasonable to investigate whether Martin's solution for the developed flow is applicable to the leakage flow.

\section{ANALYTICAL SOLUTION FOR A NEWTONIAN FLUID}

For a Newtonian fluid with a viscosity which does not depend on temperature, the coupling between the momentum and the energy equations is simple. The velocity profile may be found from the momentum equation, the result is thereafter used to solve the energy equation.

For our case of pure drag flow in the tangential direction we define a cartesian coordinate system $x, y, z$, in which $x$ is the tangential direction (making an angle $\phi$, the pitch angle, with the screw flight), $y$ is the radial direction, perpendicular to the two plates of the model and $z$ is the axial direction of the screw.

The velocities in $x, y, z$ directions are $u, v, w$ respectively. With $v=0, w=0$ and $P=$ constant, the momentum equation reduces to:

$$
\frac{\partial \tau}{\partial y}=0
$$

For a Newtonian fluid:

$$
\tau=\mu \frac{\partial u}{\partial y}
$$

And the energy equation gives:

$$
\frac{u}{a} \frac{\partial T}{\partial x}=\frac{\partial^{2} T}{\partial y^{2}}+\frac{\tau}{\lambda} \frac{\partial u}{\partial y}
$$

in which $a=\lambda / \rho c_{p}$. The boundary conditions are: $y=0$ : $u=0, T=T_{s} ; x=0: T=T_{i n} ; y=\delta: u=U_{b}, T=T_{b}$. From Eqs 1 and 2 and the boundary conditions the velocity distribution follows:

$$
u=\frac{U_{b}}{\delta} y
$$

in which $U_{b}=\pi D N$ is the velocity of the upper plate and $\delta$ is the plate separation.
With this result the energy equation becomes:

$$
\frac{u}{a} \frac{\partial T}{\partial x}=\frac{\partial^{2} T}{\partial y^{2}}+\frac{\mu}{\lambda}\left(\frac{U_{b}}{\delta}\right)^{2}
$$

This equation is made dimensionless by setting $\eta=y / \delta$, $\xi=x / \delta$ and $\theta=\left(T-T_{s}\right) /\left(T_{b}-T_{s}\right.$, ) which results in:

$$
P e \cdot \eta \cdot \frac{\partial \theta}{\partial \xi}=\frac{\partial^{2} \theta}{\partial \eta^{2}}+B r
$$

In $E q 4 P e$ is the Peclet number $P e=U_{b} \cdot \delta / a$ and $B r$ is the Brinkman number

$$
B r=\frac{\mu U_{b}^{2}}{\lambda\left(T_{b}-T_{s}\right)}
$$

For $\xi \rightarrow \infty$, the temperature is developed, there is no further change of temperature with $\xi$, consequently $(\partial \theta / \partial \xi)_{\zeta \rightarrow \infty}=0$.

Designating the developed temperature as $\theta_{\infty}, E q 4$ becomes:

$$
\frac{\partial^{2} \theta_{\infty}}{\partial \eta^{2}}=-B r
$$

with boundary conditions $\theta=0$ for $\eta=0$ and $\theta=1$ for $\eta$ $=1$.

The solution of $E q 5$ is:

$$
\theta_{\infty}=\frac{1}{2} B r \cdot \eta(1-\eta)+\eta
$$

A simplification of $E q 4$ is now possible by introducing a new variable $\theta^{*}=\theta-\theta_{\infty}$. Physically this is the deviation of local temperature from developed temperature. Because of $E q 5, E q 4$ now becomes:

$$
P e \cdot \eta \frac{\partial \theta^{*}}{\partial \xi}=\frac{\partial^{2} \theta^{*}}{\partial \eta^{2}}
$$

with boundary conditions: $\xi=0, \theta^{*}=\theta_{i n}^{*}, \eta=0, \theta^{*}=$ 0 ; and $\eta=1, \theta^{*}=0$.

The solution of this equation which may be found in most textbooks on differential equations, proceeds as follows: Let $\theta^{*}$ assume the form $\theta^{*}=E(\eta) \cdot X(\xi)$, then $\mathrm{Eq} 7$ may be written:

$$
P e \eta \cdot E \frac{\partial X}{\partial \xi}=X \cdot \frac{\partial^{2} E}{\partial \eta^{2}}
$$

Division by $\eta E X$ yields:

$$
P e \cdot \frac{1}{X} \frac{\partial X}{\partial \xi}=\frac{1}{\eta E} \frac{\partial^{2} E}{\partial \eta^{2}}=-k^{2}
$$

in which $k$ is a constant as the left hand side of the equation is a function of only $\xi$ while the right hand side is a function of $\eta$ only. Integration of the left hand side gives:

$$
X(\xi)=C_{k} \exp \left(-\frac{k^{2}}{P e} \xi\right)
$$

For the right hand side:

$$
\frac{\partial^{2} E}{\partial \eta^{2}}+k^{2} \eta E=0
$$

the substitution $\eta^{*}=\eta k^{2 / 3}$ leads to:

$$
\frac{\partial^{2} E}{\partial \eta^{* 2}}+\eta^{*} E=0
$$


for which the boundary conditions are: $\eta=0$ and $\eta=1$; both require $\theta^{*}=0$, therefore $E=0$.

Using the first boundary condition a solution is found: $E=C \cdot A i\left(\eta^{*}\right)$, in which $A i\left(\eta^{*}\right)$ is an Airy function and $C$ is a constant.

The second boundary condition requires $E$ to be equal to zero for $\eta=1$, therefore for $\eta^{*}=k^{2 / 3}$. A handbook of tabulated functions, e.g., Abramowitz \& Stegun (11), gives values of $\eta^{*}$ for which $A i\left(\eta^{*}\right)$ is zero.

A number of solutions $\eta_{i}^{*}$ is therefore possible and for each $\eta_{i}^{*}$ a value $k_{i}$ follows. The general solution is then:

$$
E=\sum_{i} C_{i} A i\left(\eta k_{i}^{2 / 3}\right)
$$

The solution of $E q 7$ is accordingly:

$$
\theta^{*}=\sum_{i} C_{k} \cdot C_{i} \cdot \operatorname{Ai}\left(\eta k_{i}^{2 / 3}\right) \cdot \exp \left(-\frac{k_{i}^{2}}{P e} \cdot \xi\right)
$$

The coefficients $C_{k} \cdot C_{i}$ may be calculated from the initial temperature profile, which must be known for this purpose, as $\theta^{*}=\theta_{i n}^{*}$ for $\xi=0$. For our purpose a knowledge of these coefficients is not strictly necessary.

For larger values of $\xi$, that is in the measure that the temperature profile develops, the first term of the series (Eq 9$)$ becomes more and more dominant. For this first term, according to the tables $\eta^{*}=2.338$, therefore $k_{1}=$ $(2.338)^{3 / 2} \approx 3.57 ; k_{1}^{2}=12.78$. For large $\xi$ however the value of $\theta^{*}$ is largely determined by the value of the exponential.

Development length is now defined as the length in flow direction over which the original value $\theta_{i n}^{*}$ decreases to $\theta^{*}=0.01 \theta_{i n}^{*}$ (the factor 0.01 is arbitrary, Yates uses 0.05). As $\theta_{i n}^{*}=\sum_{i} C_{k} \cdot C_{1} A i\left(\eta k_{i}^{2 / 3}\right)$ and for large $\xi$ only the first term, $(i=1)$ in $E q 9$, is of any importance, the condition $\theta^{*}<0.01 \theta_{i n}^{*}$ is certainly met if

$$
\exp \left(-\frac{k_{1}^{2}}{P e} \xi\right)<0.01
$$

now $0.01 \approx \exp (-4.6)$. Therefore $\xi>4.6 P e / k_{1}^{2}=$ $0.360 \mathrm{Pe}$ or roughly $3 / 8 \mathrm{Pe}$.

In a $60 \mathrm{~mm}$ extruder a value $U_{b}=0.3 \mathrm{~m} / \mathrm{sec}$ is reached at $96 \mathrm{rpm}, \delta=0.1 \mathrm{~mm}$ is a reasonable clearance and for polyethylene $a \approx 10^{-7} \mathrm{~m}^{2} / \mathrm{sec}$. These values give a Peclet number $P e=300$, from which $\xi=x / \delta>112$ or $x>$ $11.2 \mathrm{~mm}$. At a flight width of $5 \mathrm{~mm}$ and a pitch angle of $17^{\circ} 40^{\prime}$, the length of the gap in tangential direction is $L$ $=16.5 \mathrm{~mm}$ which is about 50 percent more than the development length calculated above. (With Yates' criterion $\theta^{*}<0.05 \theta_{i n}^{*}$, the result is $\xi>3 \mathrm{Pe} / k_{1}^{2}$ or $x / \delta>$ $1 / 4 P e, x>7.5 \mathrm{~mm}$ in our example.).

Unless the Peclet number is unusually high or the screw is badly worn, the temperature profile on the exit side of the flight gap may be considered fully developed for a Newtonian melt.

\section{THE NON-NEWTONIAN, TEMPERATURE DEPENDENT FLOW}

Assuming a power law dependence of viscosity on shear rate, using the lubrication approximation and $\partial P / \partial x=0$, we have the following set of equations:

$$
\begin{gathered}
\tau=\mu_{o} \exp \left\{\left(-b\left(T-T_{o}\right)\right\}\left(\frac{\partial u}{\partial y}\right)^{1-2 \alpha}\right. \\
\frac{\partial \tau}{\partial y}=0 \\
\frac{u}{a} \frac{\partial T}{\partial x}=\frac{\partial^{2} T}{\partial y^{2}}+\frac{\tau}{\lambda} \frac{\partial u}{\partial y}
\end{gathered}
$$

in which $\mu_{o}$ is the viscosity at a reference temperature $T_{o}$ and a shear rate of $1 \mathrm{sec}^{-1}$ and $\alpha$ is the power law coefficient. In dimensionless form with $u^{\prime}=u / U_{b}$; $\eta=y / \delta ; \xi=x / \delta$ and $\theta=b\left(T-T_{o}\right) / G r$ in which $G r$ is the Griffith number for the power law case $G r=$ $\mu_{o} b U_{b}^{2-2 \alpha} \delta^{2 \alpha} / \lambda$, we find for Eqs 11 and 12 the following equations:

$$
\begin{gathered}
\frac{\partial}{\partial \eta}\left\{e^{-G r \theta} \cdot\left(\frac{\partial u^{\prime}}{\partial \eta}\right)^{1-2 \alpha}\right\}=0 \\
P e u^{\prime} \frac{\partial \theta}{\partial \xi}=\frac{\partial^{2} \theta}{\partial \eta^{2}}+e^{-G r \theta}\left(\frac{\partial u^{\prime}}{\partial \eta}\right)^{2-2 \alpha}
\end{gathered}
$$

The boundary conditions are:

$$
\begin{array}{ll}
\eta=0, u^{\prime}=0, & \theta=\frac{b\left(T_{s}-T_{o}\right)}{G r}=\theta_{s} \\
\eta=1, u^{\prime}=1, & \theta=\frac{b\left(T_{b}-T_{o}\right)}{G r}=\theta_{b} \\
\xi=0, u^{\prime}=u_{i n}^{\prime}, & \theta=\theta_{i n}
\end{array}
$$

( $\theta_{\text {in }}$ being assumed known, $u_{\text {in }}^{\prime}$ follows from $E q$ 13).

These coupled differential equations must be solved numerically. We used the implicit Crank-Nicolson method (see e.g., Ref. 12) and a simple iterative process. First of all the developed temperature $\theta_{\infty}$ is calculated. This problem was solved by Martin (10) and his result was used. Then the developing temperature $\theta(\xi)$ is calculated; for $\eta$ increments of 0.05 are used, for $\xi$ the initial step is 0.01 which is increased by 2.5 percent each following step. The thermal entrance length is now defined as the length $\xi$ for which

$$
\sqrt{\frac{\int_{0}^{1}\left(\theta(\xi)-\theta_{\infty}\right)^{2} \mathrm{~d} \eta}{\int_{0}^{1}\left(\theta_{i n}-\theta_{\infty}\right)^{2} \mathrm{~d} \eta}}<0.01
$$

This is a relative criterion which comes close to the criterion $\theta^{*} / \theta_{i n}^{*}<0.01 \perp\left(\theta^{*}=\theta-\theta_{\infty} ; \theta_{i n}^{*}=\theta_{i n}-\theta_{\infty}\right)$ used in the analytical (Newtonian) solution where no further information on $\theta_{i n}$ was required.

In the numerical solution here $\theta_{i n}$ must be given explicitly and the absolute difference between this initial temperature profile and the developed profile (expressed as the sum of squared deviations) can be found by numerical integration. By taking one percent of this value the criterion for $\theta(\xi)$ is known and the entrance length follows.

The most important objective here is the test of the applicability of the analytical solution $L / \delta=3 / 8 P e$, under a number of different conditions. According to the Eqs 13 and 14 the temperature development is completely determined by (a) the Peclet number, (b) the Griffith number, (c) the power law coefficient $\alpha$ and by the boundary conditions from $E q 15$, (d) $\theta_{b}$, (e) $\theta_{s}$ and (f) $\theta_{i n}$. By varying them independently we can scan behavior of the solution over a relatively wide range. 


\section{RESULTS OF THE NUMERICAL CALCULATIONS}

As basic conditions we used:

$$
\begin{aligned}
T_{b}=190\left({ }^{\circ} \mathrm{C}\right), T_{s}=180\left({ }^{\circ} \mathrm{C}\right), 140\left({ }^{\circ} \mathrm{C}\right)<T_{\text {in }}<230\left({ }^{\circ} \mathrm{C}\right) \\
b=0.012\left({ }^{\circ} \mathrm{C}^{-1}\right), \alpha=0.25(-), \\
\mu_{o}=6500\left(\mathrm{Nsec} / \mathrm{m}^{2}\right), T_{o}=185\left({ }^{\circ} \mathrm{C}\right)
\end{aligned}
$$

$\omega=10(\mathrm{rad} / \mathrm{sec}), \delta=10^{-4}(\mathrm{~m})$,

$$
D=6.10^{-2}(\mathrm{~m}), a=10^{-7}\left(\mathrm{~m}^{2} / \mathrm{sec}\right)
$$

$\lambda=0.18\left(\mathrm{~W} / \mathrm{m}^{\circ} \mathrm{C}\right)$, so $P e \approx 300, G r \approx 0.5$

- The entrance length is linearly dependent on the Peclet number in all cases.

- The value of the power law coefficient $\alpha$ does not influence the entrance length very much; rather high values of $\alpha$ give values of the calculated entrance lengths which are only $\dot{a}$ few percent lower than for $\alpha=0$. The Newtonian case therefore errs slightly on the safe side.

- With fixed boundary conditions $\theta_{b}$ and $\theta_{s}$ (and a fixed Peclet number, $P e=300$ and $\alpha=0.25$ ) a variation of the entrance temperature $\theta_{\text {in }}$ between rather low and high values, gives, for different values of the Griffith number, the results as shown in Fig. 1. Apparently the entrance length reaches a minimum value when $\theta_{i n_{-}}$is somewhat higher than $\theta_{b}$ and approaches the average $\theta_{\infty}$. This is not surprising, because for this condition the adaptation required is minimal. (Note: In this example and in the following $\theta_{i n}$ represents a uniform entrance

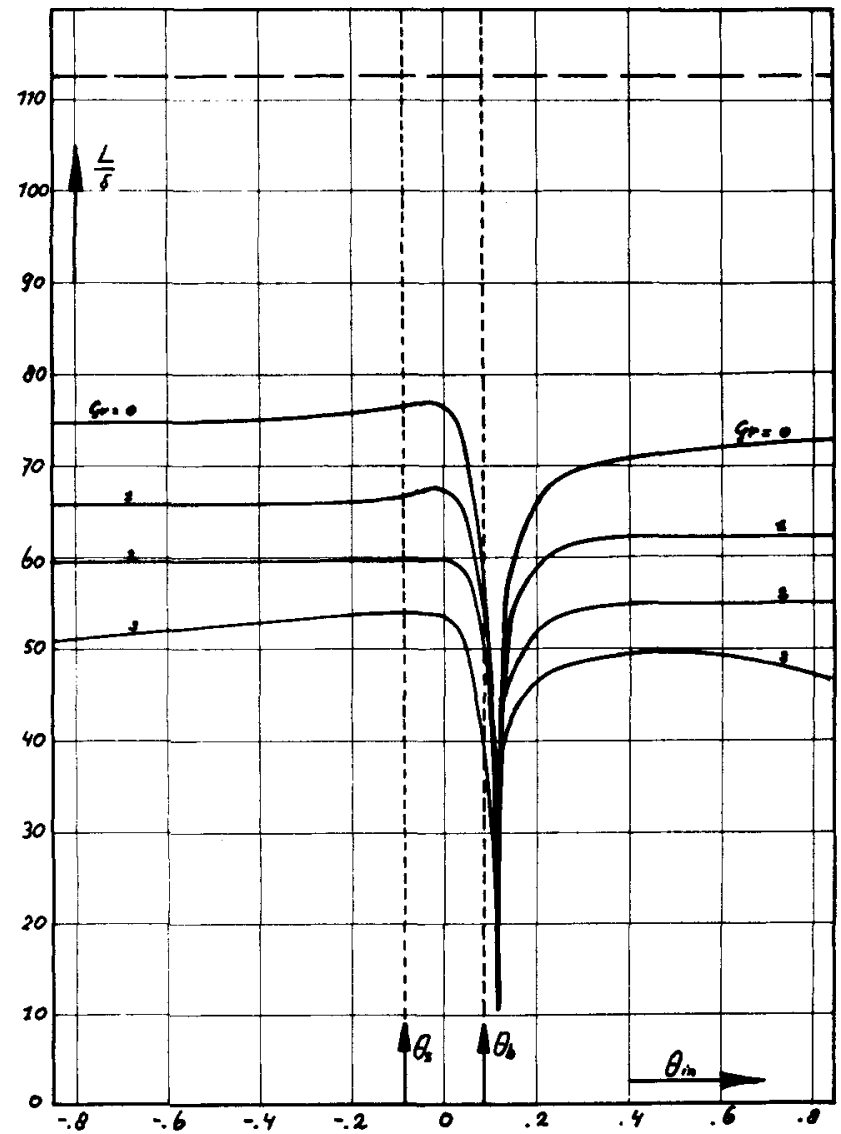

Fig. I. Thermal entrance length as a function of entrance temperature, for different values of the Griffith-number. Fixed boundary conditions $\theta_{\mathrm{s}}$ and $\theta_{b}, \alpha=0.25, P e=300 ;$ - : line $L / \delta$ $=3 / 8 \mathrm{Pe}$ temperature. In the preceding theory $\theta_{i n}$ may be any function of $\eta$ and is not necessarily uniform.)

- With fixed boundary condition $\theta_{b}$, again $P e=300$, $\alpha=0.25$ and changing $\theta_{s}$ and $\theta_{i n}$ (so that $\theta_{s}=\theta_{i n}$ ) from relatively low values (as could occur in the beginning of the melting zone) to high values (e.g., in the metering zone of an extruder with a neutral screw) we find similar results as shown in Fig. 2. Remarkable here is the different influence of the Griffith number below and above the minimum point.

- In all cases, the calculated entrance length is clearly less than that of the analytical solution (The only exception is found in Fig. 2 for high Griffith numbers and very high $\theta_{i n}=\theta_{s}$. Remembering that $\theta=b(T-$ $\left.T_{o}\right) / G r$, so $T=G r \cdot \theta / b+T_{o}$, it follows that for normal extruders these values of $G r$ and $\theta$ lead to unrealistically high temperatures.)

- The maximum absolute temperature differences $(T$ $\left.-T_{\infty}\right)$, at the place where the entrance length was reached according to the relative criterion, was of the order of $0.2-0.3^{\circ} \mathrm{C}$.

\section{CONCLUSIONS}

The entrance lengths calculated for a non-Newtonian fluid with temperature dependent viscosity, remain, under the most different conditions, below the result of the Newtonian analysis:

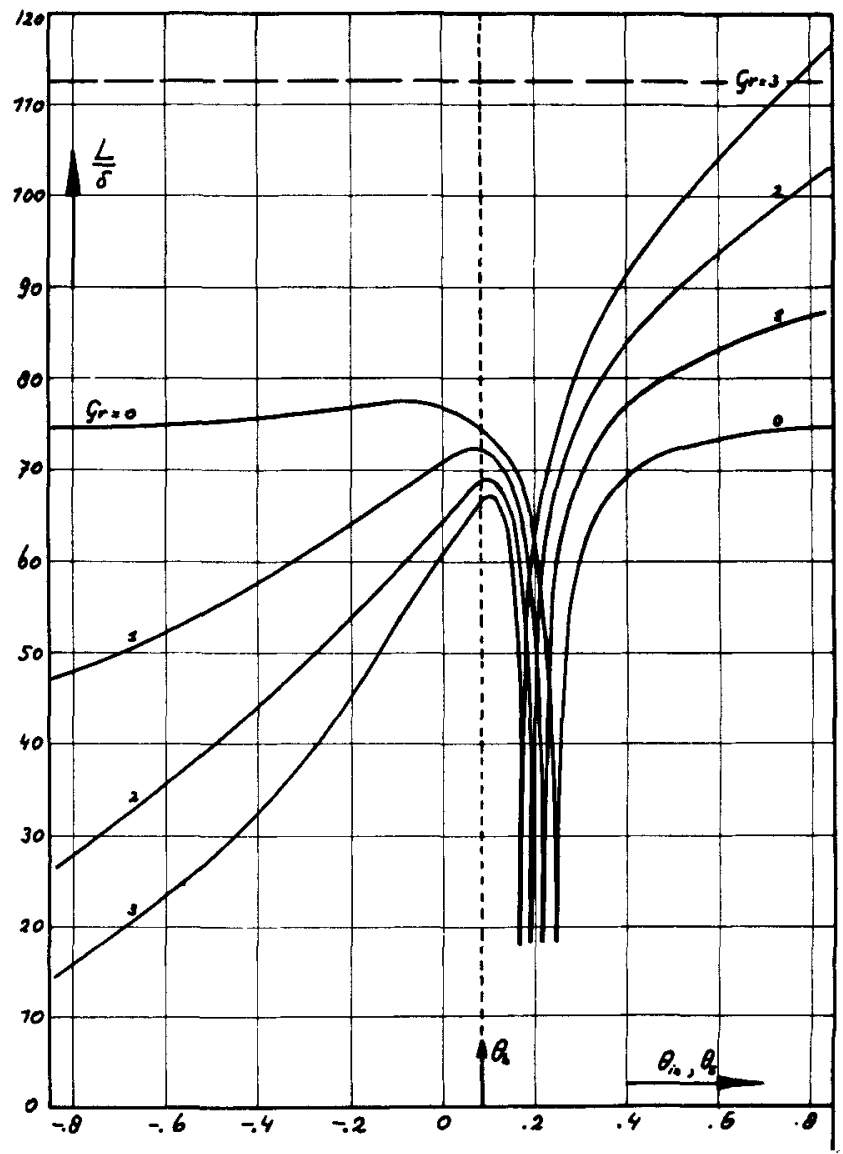

Fig. 2. Thermal entrance length as a function of entrance and screw temperature $\theta_{s}=\theta_{i n}$, for different values of the Griffithnumber. Fixed boundary condition $\theta_{b}, \alpha=0.25, \mathrm{Pe}=300$; - line $L / \delta=3 / 8 \mathrm{Pe}$. 


$$
\frac{L}{\delta} \approx \frac{3}{8} \cdot P e
$$

which may therefore be used as an upper bound.

The numerical calculations show that in most cases, and certainly for $T_{i n}<T_{b}$, we may use:

$$
\frac{L}{\delta} \approx \frac{1}{4} \cdot P e
$$

The maximum absolute temperature difference between the developing flow and the developed flow at the entrance length thus calculated is of the order of a few tenths of one degree. This means that in extruders in reasonable condition where the flight clearance $\delta$ is not too big, the thermal development length will be smaller than the available gap length in tangential direction. The convection terms in the energy equation in the calculation of this flow may therefore be neglected and Martin's results (10) can be applied to find the temperature and velocity distributions of the flow at the exit of the leakage gap in all the zones of an extruder where melt flow is present.

\section{NOMENCLATURE}

$\begin{array}{ll}a, \mathrm{~m}^{2} / \mathrm{sec} & =\text { Thermal diffusivity coefficient } \\ \mathrm{Ai}(\eta) & =\text { Airy function } \\ b,{ }^{\circ} \mathrm{C}-1 & =\text { Temperature coefficient of viscosity } \\ \mathrm{Br} & =\text { Brinkman number } \\ \mathrm{C}, C_{i}, C_{k} & =\text { Constants introduced in and after } E q \\ & 8 \\ c_{p}, \mathrm{~J} / \mathrm{kg}{ }^{\circ} \mathrm{C} & =\text { Specific heat } \\ D, \mathrm{~m} & =\text { Diameter of the barrel } \\ E(\eta) & =\text { Function introduced after } E q 7 \\ G r & =\text { Griffith number } \\ k, k_{i} & =\text { Constants introduced in } E q 8 \\ L, \mathrm{~m} & =\text { Length of screw flight in tangential } \\ & \text { direction } \\ N, \mathrm{sec}-1 & =\text { Screw speed } \\ P, \mathrm{~N} / \mathrm{m}^{2} & =\text { Pressure } \\ P e & =\text { Peclet number } \\ T,{ }^{\circ} \mathrm{C} & =\text { Temperature } \\ T_{b},{ }^{\circ} \mathrm{C} & =\text { Barrel temperature } \\ T_{i n},{ }^{\circ} \mathrm{C} & =\text { Entrance temperature } \\ T_{s},{ }^{\circ} \mathrm{C} & =\text { Screw temperature } \\ T_{b},{ }^{\circ} \mathrm{C} & =\text { Reference temperature } \\ u, \mathrm{~m} / \mathrm{sec} & =\text { Velocity in tangential } x \text {-direction } \\ u^{\prime} & =\text { Dimensionless velocity }\end{array}$

$$
\begin{aligned}
& u_{\text {in }}^{\prime} \quad=\text { Dimensionless entrance velocity } \\
& U_{b}, \mathrm{~m} / \mathrm{sec} \quad=\text { Barrel velocity } \\
& v, \mathrm{~m} / \mathrm{sec} \quad=\text { Velocity in radial } y \text {-direction } \\
& w, \mathrm{~m} / \mathrm{sec} \quad=\text { Velocity in axial } z \text {-direction } \\
& X(\xi) \quad=\text { Function introduced after } E q 7 \\
& x, \mathrm{~m} \quad=\text { Tangential direction } \\
& y, \mathrm{~m} \quad=\text { Radial direction } \\
& z, \mathrm{~m} \quad=\text { Axial direction } \\
& \alpha \quad=\text { Power law coefficient } \\
& \delta, \mathrm{m} \quad=\text { Height of leakage gap } \\
& \eta, \eta^{*} \quad=\text { Dimensionless radial direction } \\
& \theta \quad=\text { Dimensionless temperature } \\
& \theta_{b} \quad=\text { Barrel temperature } \\
& \theta_{\text {in }} \quad=\text { Entrance temperature } \\
& \theta_{s} \quad=\text { Screw temperature } \\
& \theta_{o} \quad=\text { Reference temperature } \\
& \theta^{*} \quad=\text { Difference between local and fully } \\
& \theta_{\infty} \quad=\text { Fully developed temperature } \\
& \lambda, \mathrm{W} / \mathrm{m}{ }^{\circ} \mathrm{C}=\text { Thermal conductivity of melt } \\
& \mu, \mathrm{Nsec} / \mathrm{m}^{2}=\text { Viscosity } \\
& \mu_{\theta}, \mathrm{Nsec}^{1-2 \alpha} / \mathrm{m}^{2}=\text { Material constant in constitutive } \\
& \xi \quad=\text { Dimensionless tangential direction } \\
& \rho, \mathrm{kg} / \mathrm{m}^{3} \quad=\text { Density of melt } \\
& \tau, \mathrm{N} / \mathrm{m}^{2} \quad=\text { Shear stress }
\end{aligned}
$$

\section{REFERENCES}

1. Z. Tadmor, Polym. Eng. Sci, 6, 185 (1966).

2. J. Shapiro, Melting in Plasticating Extruders, Ph.D. Thesis, Cambridge, England (1971).

3. I. R. Edmondson and R. T. Fenner, Polymer, 16, 49(1975).

4. B. Yates, Temperature Development in Single Screw Extruders, Ph.D. Thesis, Cambridge, England (1968).

5. W. D. Mohr and R. S. Mallouk, Ind. Eng. Chem., 15, 383 (1959).

6. J. F. Ingen Housz, Plastverarbeiter, 26, 573 (1975).

7. E. A. Muyderman, "Spiral Groove Bearings," Philips Technical Library, Eindhoven (1966).

8. D. B. Holmes, "Experimental Studies of Laminar Flow in Ducts," Ph.D. Thesis, Delft, Netherlands (1967).

9. K. Hayashida, Rheol. Acta, 2, 261 (1962).

10. B. Martin, Int. J. Non-Linear Mech., 2, 285 (1967).

11. M. Abramowitch and I. A. Stegun, "Handbook of Mathematical Functions," Dover Publ., Inc., New York.

12. G. E. Forsythe and W. R. Wasow, "Finite Difference Methods for Partial Differential Equations," John Wiley \& Sons, Inc. New York. 